\title{
Global ergodic capacity Closed-Form Expression of Coexisting DVB-LTE-Like Systems
}

Hiba BAWAB, Philippe MARY, Jean François HELARD, Youssef NASSER, Oussama BAZZI

IETR -INSA of Rennes 


\section{Plan}

- Introduction

- Convergence between DVB \& LTE

- System and signal model

- Global ergodic capacity

- Simulations

- Performance results \& discussion

- Conclusions \& Future works 


\section{Introduction-context}

- Major issue: spectrum congestion.

- Possibility to use the separated band or reuse the same band.

- Requisition of multimedia services: Internet, data sharing, TV, etc.

- Future broadcasting networks (DVB-NGH,...): necessary component of the future usage. 


\section{Introduction-Related works}

- Adaptive resource management : secondary and primary networks.

- Context of coexistence DVB(Digital Video Broadcasting) LTE(Long Term Evolution ).

- adjacent frequency bands with same given territory.

- same frequency bands with neighboring regions.

- Monte Carlo simulation,...

- Interested parameter: Protection distance.

- No recent works: ergodic capacity of full cross interfering links. 


\section{Convergence between DVB \& LTE}

- Problem: LTE small cells into a DVB large cell.

- DVB and LTE interfere on each other.

- Useful for operators.

- Goal: Derive global ergodic capacity

- Rayleigh fading links

- evaluation of the global data rate of the DVB and LTE.

- evaluation of the influence of system parameters (power, distance) on data rates. 


\section{System and signal model}

\begin{tabular}{|c|c|c|}
\hline & ymbols & Notations \\
\hline & BCT & Broadcast transmitter \\
\hline & BCR & Broadcast receiver \\
\hline & BS & Base station \\
\hline & MS & Mobile Station \\
\hline & $g$ & $\begin{array}{c}\text { Channel coefficient } \\
\text { between BCT-BCR (Rayleigh) }\end{array}$ \\
\hline & $h_{k}$ & $\begin{array}{c}\text { Channel coefficient } \\
\text { between BS-MSk (Rayleigh) }\end{array}$ \\
\hline & $q_{b k}$ & $\begin{array}{c}\text { Channel coefficient } \\
\text { between BCT-MSk } \\
\text { (Rayleigh) }\end{array}$ \\
\hline & $\mathrm{uk}_{\mathrm{k}}$ & $\begin{array}{l}\text { Channel coefficient } \\
\text { between BS-BCR (Rayleigh) }\end{array}$ \\
\hline \multicolumn{3}{|c|}{$y_{k}=\sqrt{P_{k} l_{k}} h_{k} x_{k}+\sum_{b=1} \sqrt{P_{b} l_{1}} q_{b k} x_{b}+n_{k}$} \\
\hline$y_{b}$ & $=V$ & $\overline{l_{b}} g x_{b}+\alpha \sqrt{P_{k} l_{2}} u_{k} x_{k}+n_{b}$ \\
\hline
\end{tabular}

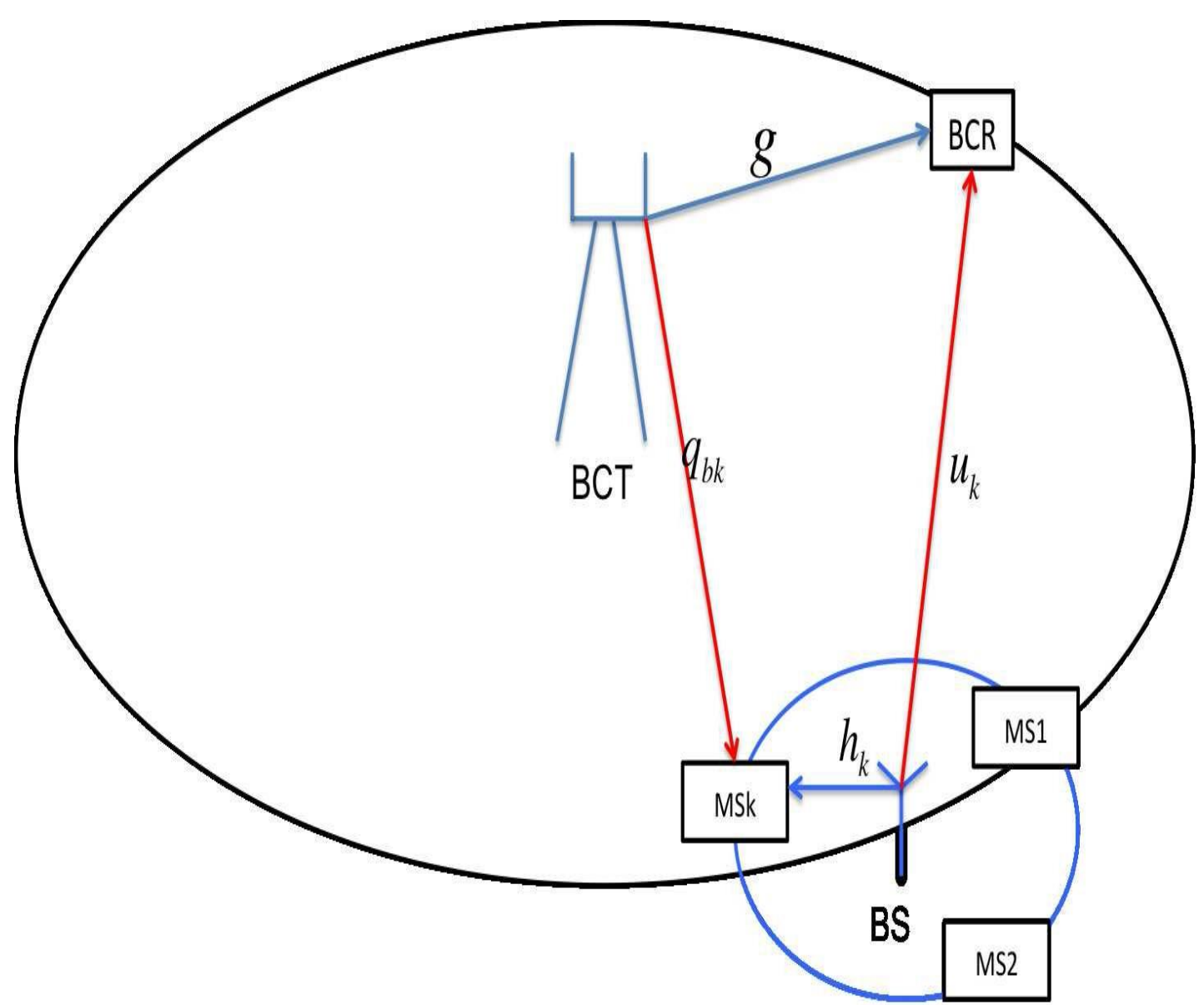

Fig.1: System model 


\section{Signal model-subcarrier}

- LTE subcarrier is more larger than DVB subcarrier.

- Subcarrier of LTE signal $=N *$ subcarrier of DVB signal

- $N>1$

- Subcarrier DVB signal $=\alpha *$ Subcarrier of LTE signal

- $\propto=1 / N$

In our study:

$\mathrm{N}=15$

$\propto=1 / 15$

LTE subcarrier

DVB subcarriers

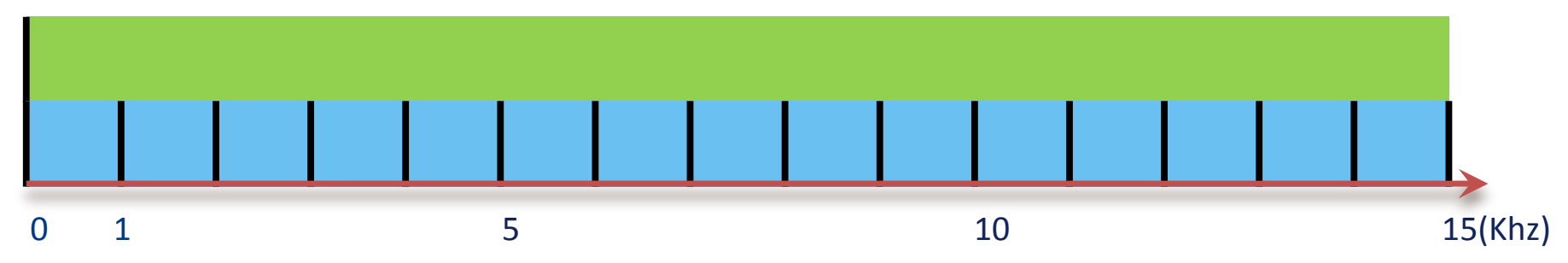




\section{Global ergodic capacity}

- Two steps:

- SIR distribution

- Global capacity expression

- Derive ergodic capacity received at BCR \& MS-k.

- Sum of two capacities.

- Bandwidth: broadcast network bandwidth 


\section{SIR distribution}

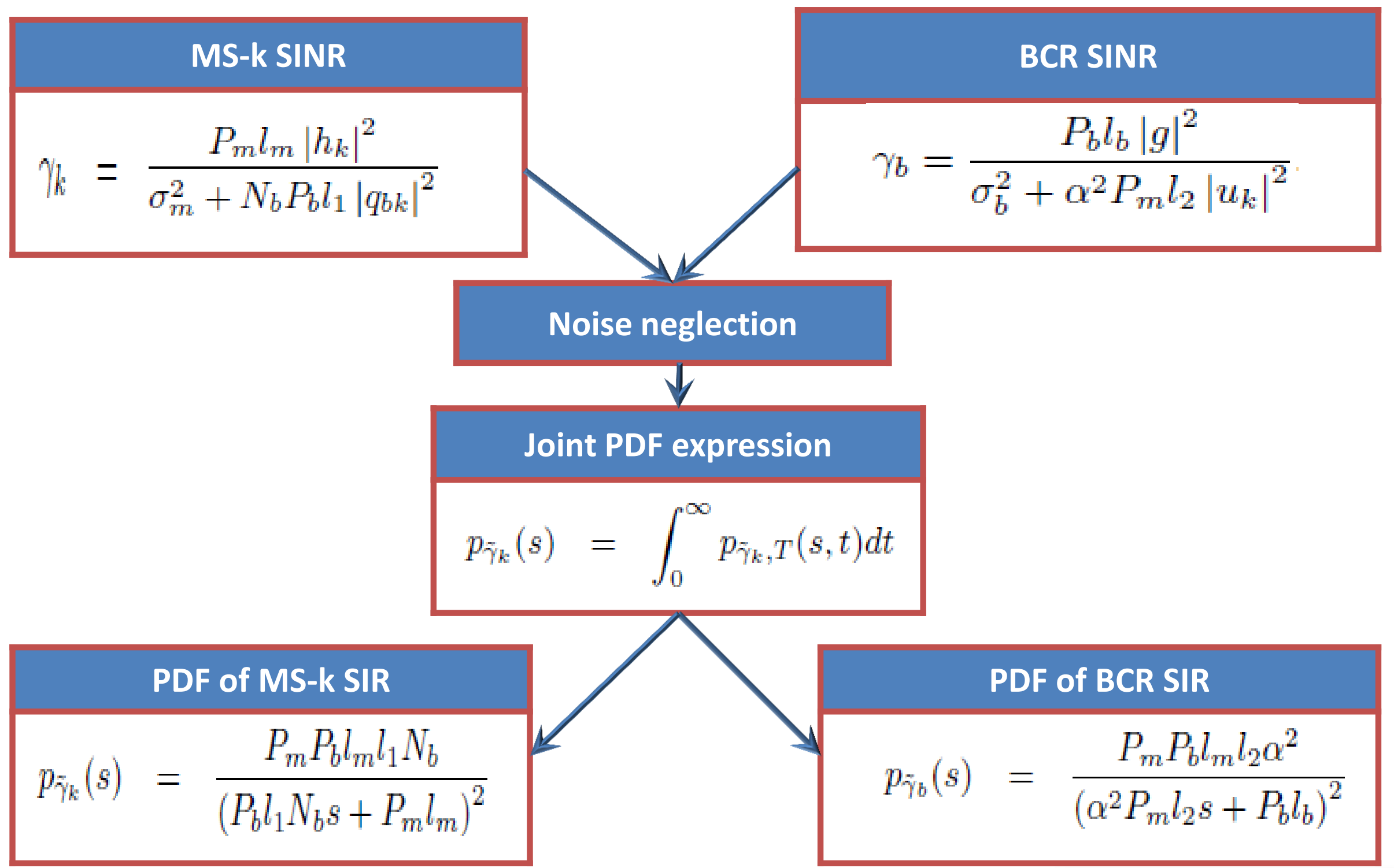




\section{Global capacity expression}

$$
\begin{gathered}
C_{\text {global }}=\mathbb{E}_{h_{k}, q_{b k}} \log _{2}\left(1+\gamma_{k}\right)+\mathbb{E}_{g, u_{k}} \log _{2}\left(1+\gamma_{b}\right) \\
C_{\text {global }}=\mathbb{E}_{\tilde{\gamma}_{k}} \log _{2}\left(1+\tilde{\gamma}_{k}\right)+\mathbb{E}_{\tilde{\gamma}_{b}} \log _{2}\left(1+\tilde{\gamma}_{b}\right)
\end{gathered}
$$

Global ergodic capacity derived

$$
C_{\text {global }}(\text { bits } / s)=W_{b}\left(\frac{P_{m} l_{m}}{N_{b} P_{b} l_{1}-P_{m} l_{m}} \log _{2} \frac{N_{b} P_{b} l_{1}}{P_{m} l_{m}}+\frac{P_{b} l_{b}}{\alpha^{2} P_{m} l_{2}-P_{b} l_{b}} \log _{2} \frac{\alpha^{2} P_{m} l_{2}}{P_{b} l_{b}}\right)
$$




\section{Simulation parameters}

- Apply realistic values of DVB \& LTE network parameters:

- Path loss attenuation factor: 2.5 in DVB, 3 in LTE

- DVB bandwidth : $W b=6.8 \mathrm{MHz}$

- Maximum total broadcast power=10 KW: Pbmax $=1,47 \mathrm{~W}$

- Maximum total BS power=50 W: Pmmax=0,09W

- DVB cell radius $=100 \mathrm{Km}$

- LTE cell radius $=1 \mathrm{Km}$

- Distance BCT-MS=100Km

- Distance BS-BCR: $d$

- Interested parameter to study 


\section{Global capacity Vs broadcast capacity}

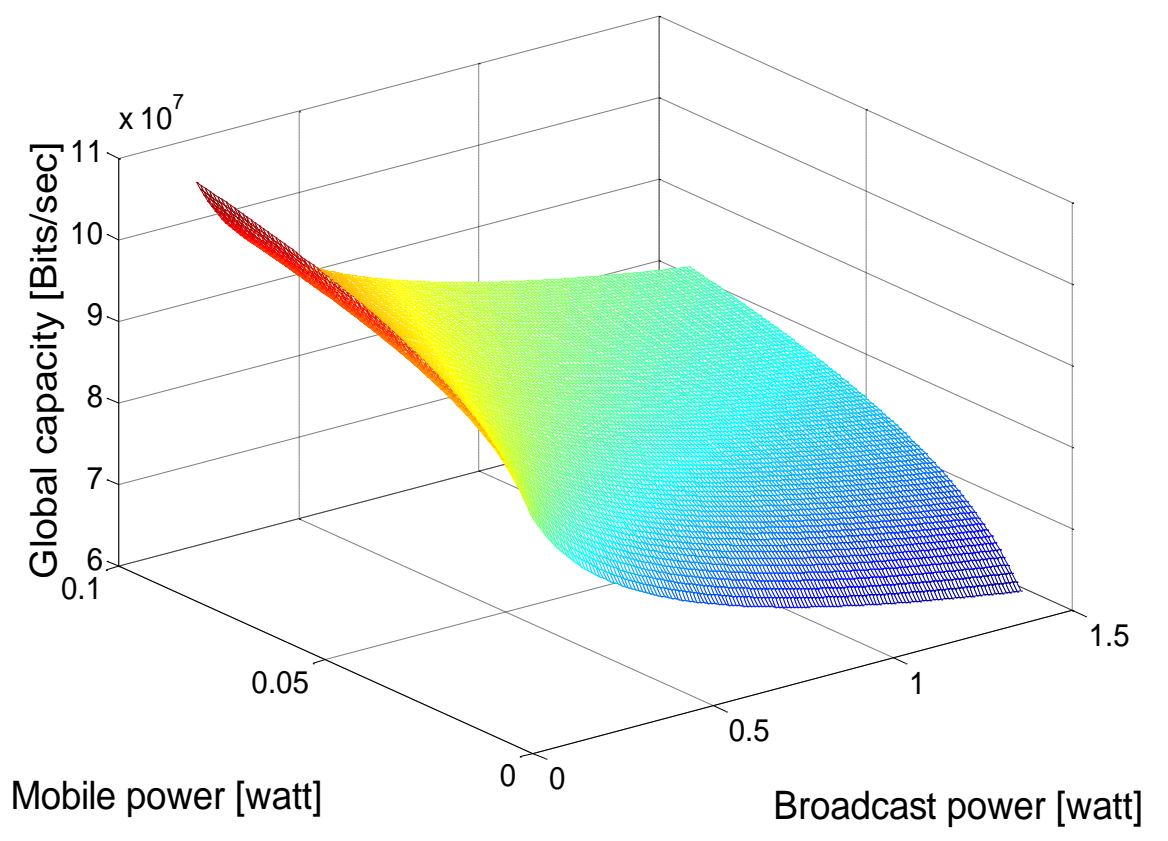

Fig.2: Global capacity in function of $\mathrm{Pm}_{\mathrm{m}}$ and $\mathrm{Pb}$

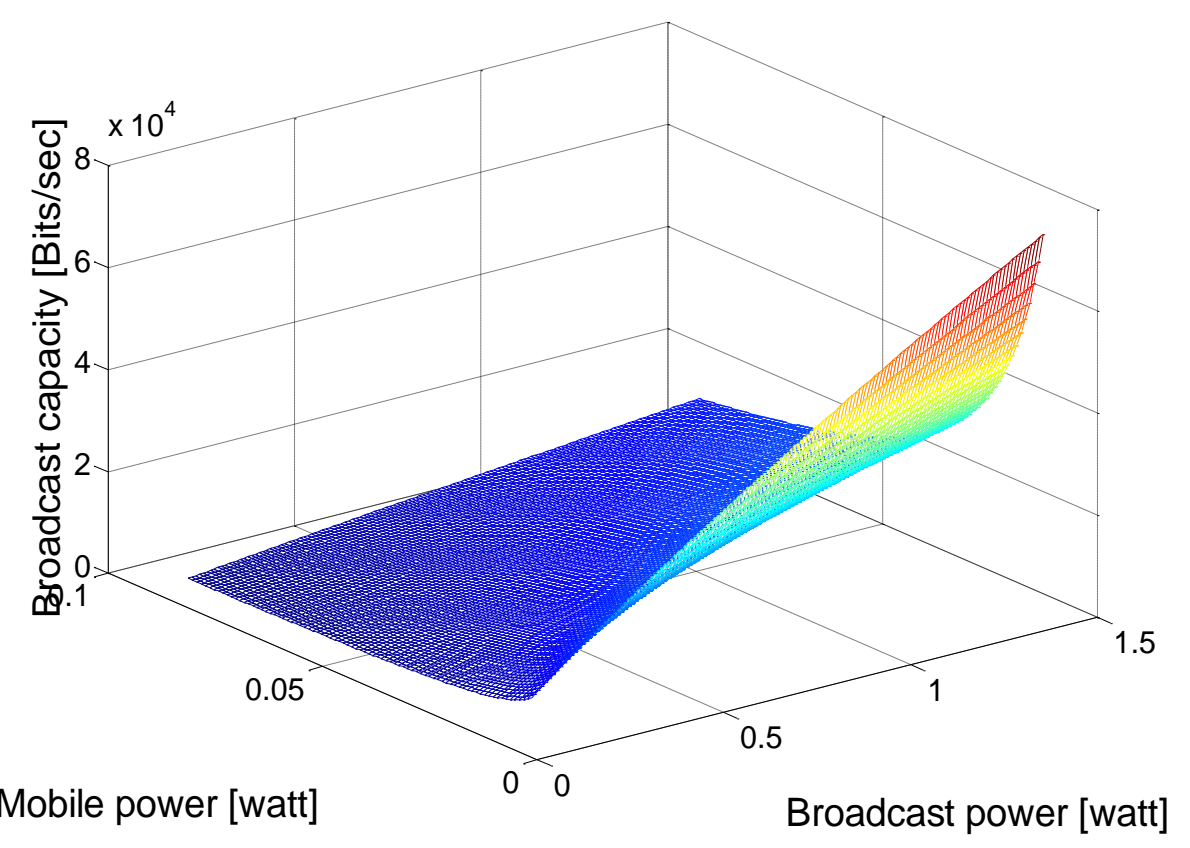

Fig.3: Broadcast capacity in function of $\mathrm{Pm}_{\mathrm{m}}$ and $\mathrm{Pb}$ 


\section{Global capacity Vs Broadcast capacity}

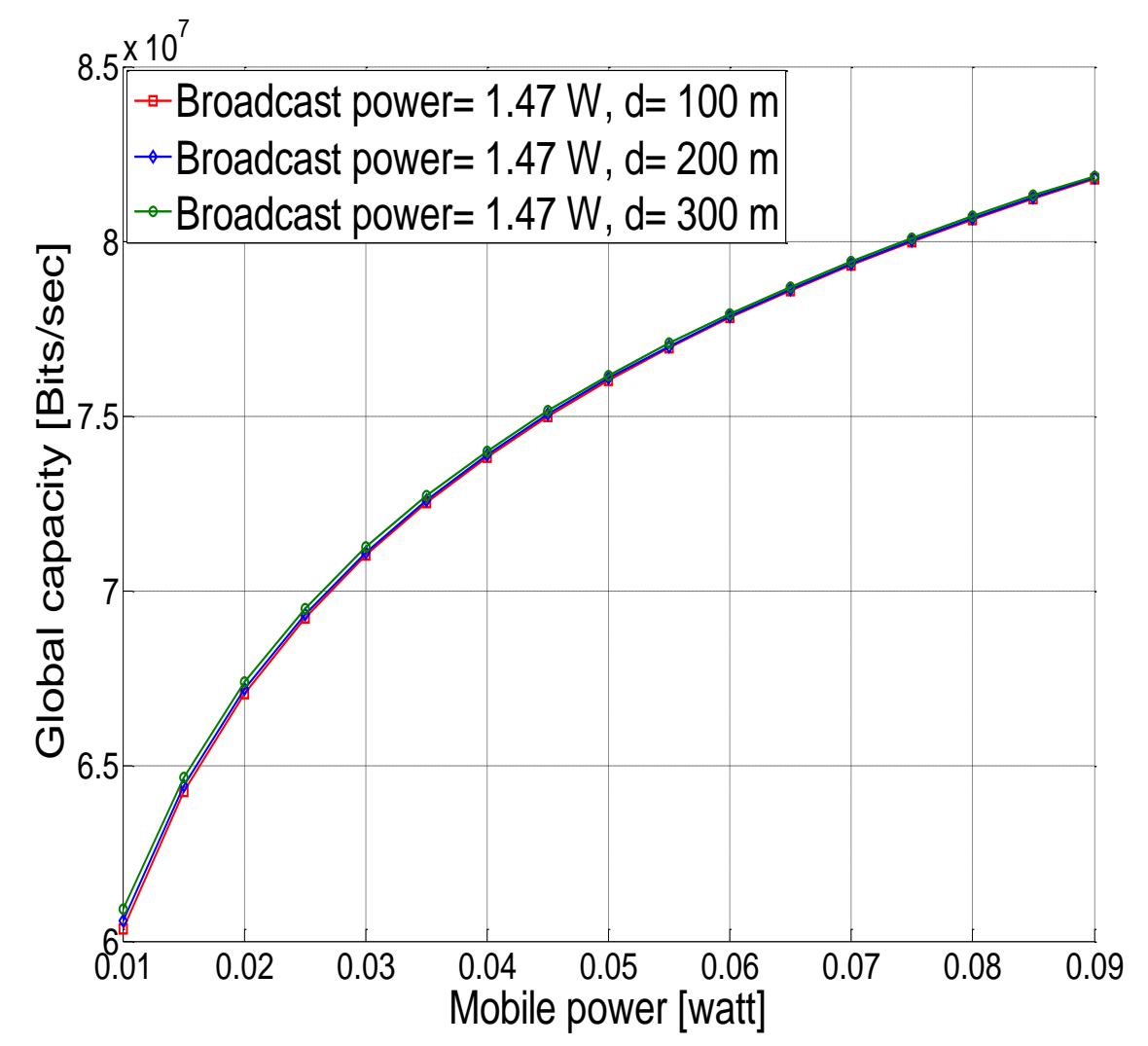

Fig.4: Global capacity in function of $P_{m}$, with $d=100,200,300 m$

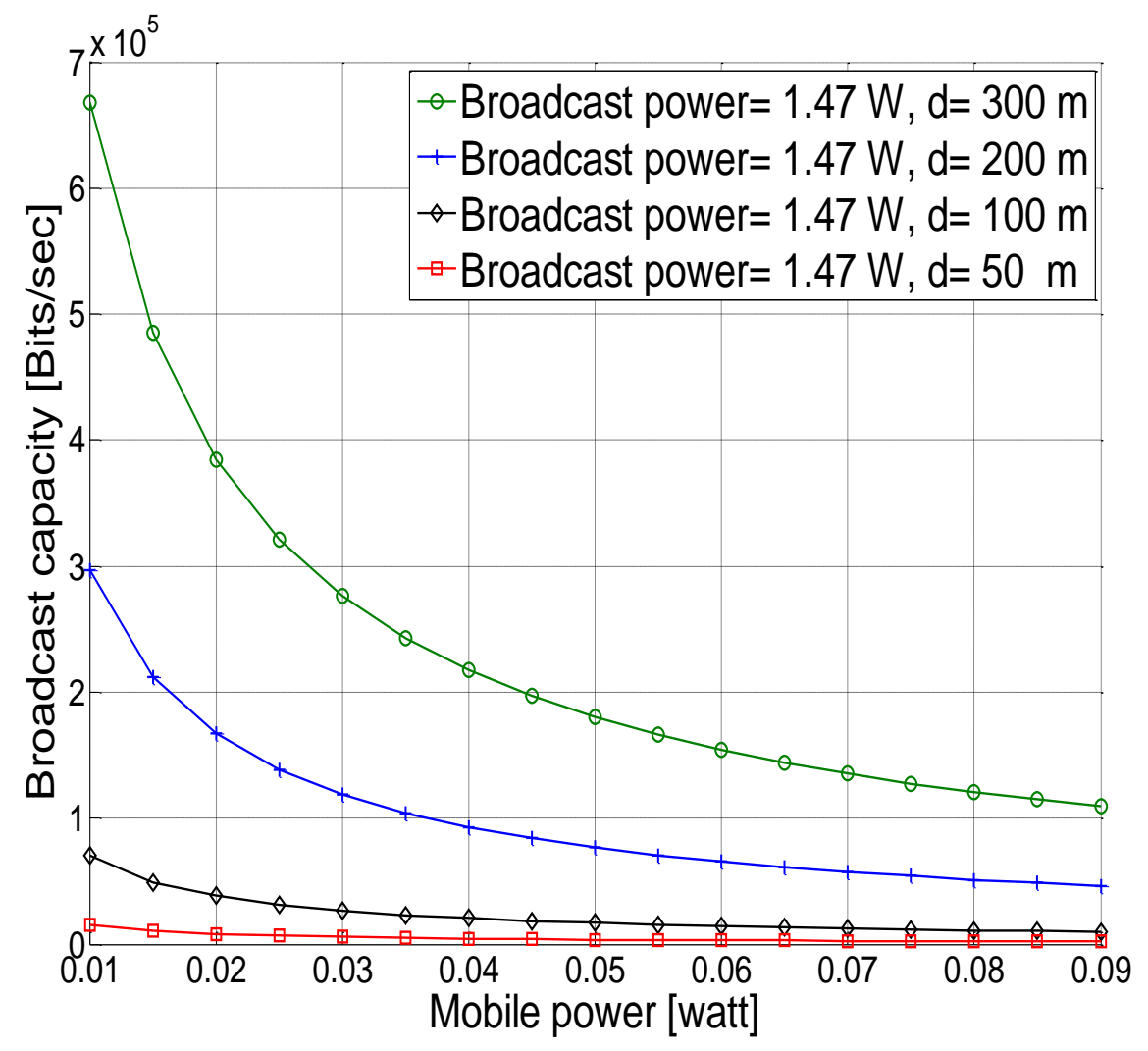

Fig.5: Broadcast capacity in function of $\mathrm{Pm}$, with $\mathrm{d}=50,100,200,300 \mathrm{~m}$ 


\section{Conclusions \& Future works}

- Conclusions :

- Derivation of the global ergodic capacity

- Dependence of the broadcast signal reception on $\mathrm{d}$.

- Global capacity: not a convex function of Pm and Pb.

- Future work:

- Broadcast and mobile capacities are both opposite objectives.

- Guarantee QOS.

- Further work: the multi objectives maximization problem.

- Usefulness of global ergodic capacity expression derived. 


\section{Thanks}

This work is supported by the French ANR project "M3" (Mobile Multimedia) and the Lebanese National Council for Scientific Research.

Thank you $(;$ 